6. Araujo I, Escribano P, Lopez-Gude MJ, et al. Giant pulmonary artery aneurysm in a patient with vasoreactive pulmonary hypertension: a case report. BMC Cardiovasc Disord. 2011;11:1-5.

7. Abdel-Rauf Z, Abdulhamid G. Idiopathic pulmonary artery aneurysm detected with multidetector computed tomography: a rare but potentially lethal vascular abnormality. IMAJ. 2011;13:581-2.

8. Shankarappa RK, Nagaraja DM, Chandrasekaran D, et al. Giant pulmonary artery aneurysm secondary to primary pulmonary hypertension. Texas Heart Inst J. 2010;37:244-5.

9. Senbaklavaci O, Kaneko Y, Bartunek A, et al. Rupture and dissection in pulmonary artery aneurysms: incidence, cause, and treatment-review and case report. J Thorac Cardiovasc Surg. 2001;121:1006-8.

10. Priya J, Sanjiv S, Rajnish J, et al. Transcatheter treatment of pulmonary artery pseudoaneurysm using a PDA closure device. Diagn Interv Radiol. 2011;17:92-4.
Víctor Hugo Contreras-Gutiérrez*, Rodolfo Castaño-Guerra

Departamento de cardiología del Hospital General de México, Ciudad de México, Mexico

* Corresponding author at: Dr Balmis 102, Colonia doctores, delegación Cuauhtémoc, Ciudad de México, CP 06720, Mexico.

E-mail address: victorcontrerasdr@hotmail.es

(V.H. Contreras-Gutiérrez).

http://dx.doi.org/10.1016/j.acmx.2017.01.002

1405-9940/

(c) 2017 Instituto Nacional de Cardiología Ignacio Chávez. Published by Masson Doyma México S.A. This is an open access article under the CC BY-NC-ND license (http: / / creativecommons.org/licenses/ by-nc-nd/4.0/).

\section{Síndrome del seno carotídeo tras endarterectomía carotídea}

\section{Carotid sinus syndrome after carotid endarterectomy}

\section{Introducción}

La endarterectomía carotídea es el tratamiento de elección de los pacientes con estenosis carotídea significativa. La incidencia de bradiarritmias en el postoperatorio precoz es frecuente si bien, estas son transitorias y de buen pronóstico, siendo anecdótica la necesidad de estimulación cardiaca permanente.

El síndrome del seno carotídeo cursa con síncopes con respuesta cardioinhibidora, vasodepresora o mixta. El tratamiento de las formas cardioinhibidoras es el implante de un marcapasos.

Presentamos el caso de un varón que comenzó con síncopes recurrentes en el postoperatorio de endarterectomía carotídea y al que se diagnosticó de síndrome del seno carotídeo.

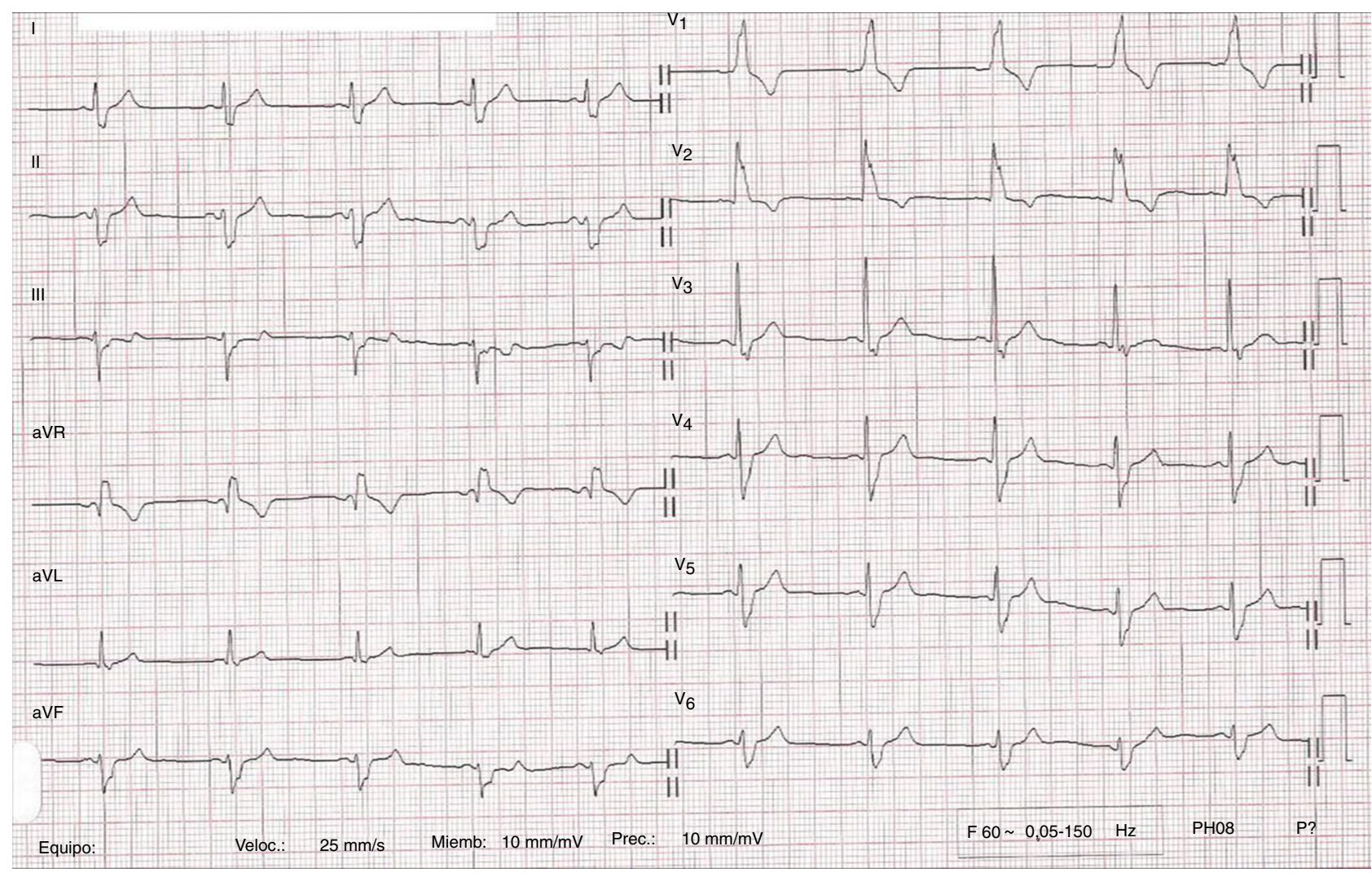

Figura 1 ECG de 12 derivaciones del paciente en el que se comprueba ritmo sinusal con bloqueo bifascicular. 

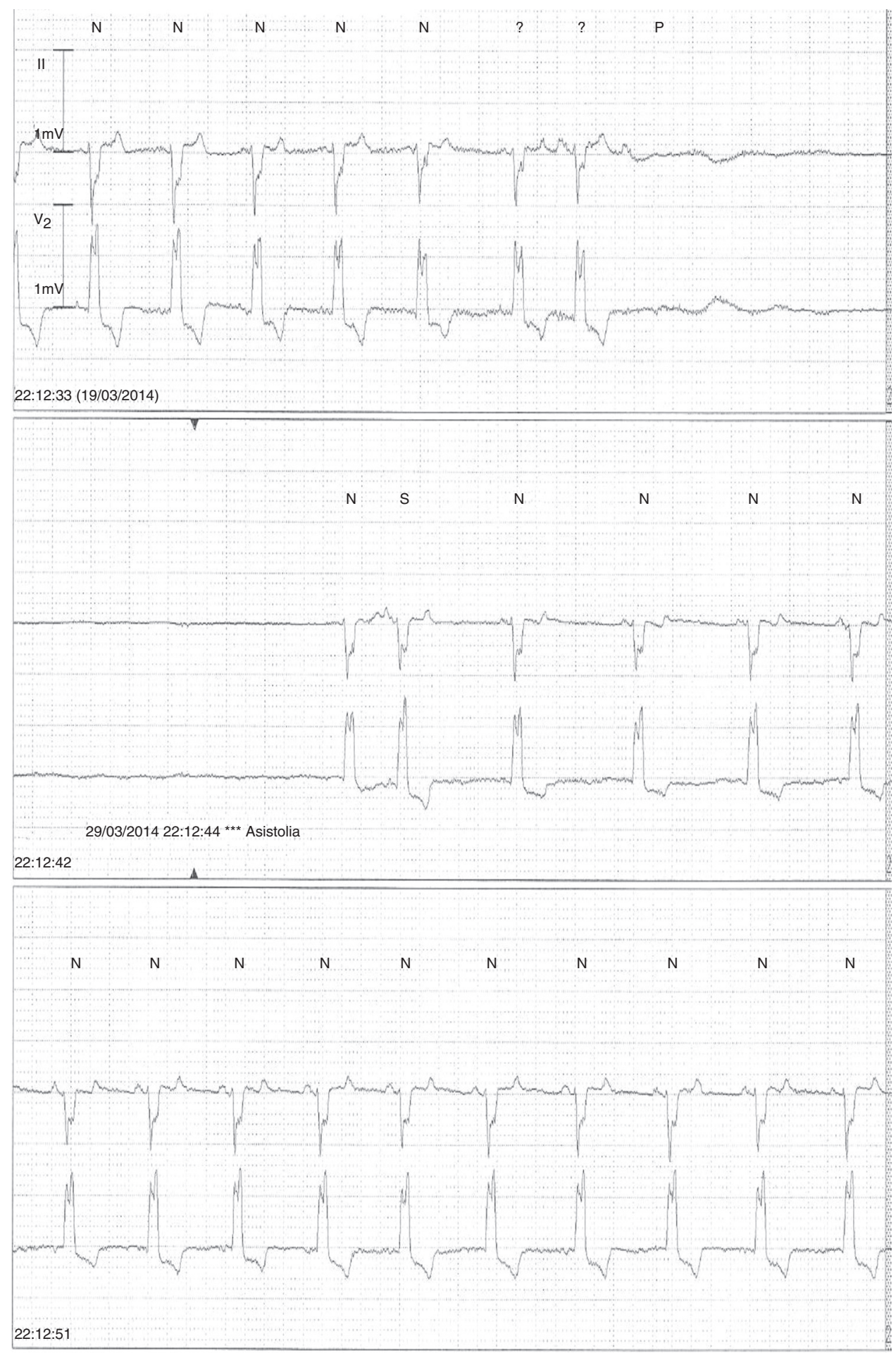

Figura 2 Registro de ECG en telemetría durante un episodio de presíncope en el que se objetiva asistolia por paro sinusal.

\section{Presentación del caso}

Varón de 59 años con antecedentes de diabetes mellitus tipo 2, hipertensión arterial, hipertrigliceridemia y estenosis significativa (85\%) asintomática de la arteria carótida derecha. Llevaba tratamiento con metformina, clopidogrel, fluvastatina, valsartán y amlodipino, y se le había practicado una endarterectomía carotídea derecha 2 semanas antes. Consultaba porque, desde la intervención quirúrgica, presentaba presíncopes y síncopes casi diarios, y muy mal control de la tensión arterial con oscilaciones frecuentes (hipertensión e hipotensión). 
La exploración física cardiaca era normal. El ECG mostraba un ritmo sinusal a $70 \mathrm{lpm}$ con bloqueo completo de rama derecha y bloqueo de la subdivisión anterior (fig. 1). El masaje del seno carotídeo izquierdo no produjo respuesta patológica, pero el masaje del seno carotídeo derecho produjo una caída de la tensión arterial sistólica de unos $40 \mathrm{mmHg}$ con sensación de mareo, aunque sin síncope. La ecocardiografía transtorácica objetivaba, como único hallazgo, hipertrofia concéntrica ligera del ventrículo izquierdo. El test de atropina fue normal (taquicardización sinusal rápida hasta alcanzar 120 (pm).

Dada la aparición postoperatoria de los síntomas, su presentación casi diaria y la existencia de un bloqueo bifascicular en la conducción intraventricular, se le recomendó ingreso hospitalario para monitorización continua de tensión arterial y ritmo cardiaco. A las pocas horas del ingreso se pudo documentar la aparición, durante la vigilia, de frecuentes asistolias sintomáticas debidas a paro sinusal (fig. 2).

Por dicho motivo se le indicó el implante de un marcapasos definitivo bicameral que se llevó a cabo sin incidencias. Modo de estimulación AAIR-DDDR con frecuencia mínima de estimulación 60 lpm, máxima de 145 lpm e intervalos AV sensado $160 \mathrm{~ms}$ y estimulado $200 \mathrm{~ms}$.

Desde el implante del marcapasos, la sintomatología del paciente desapareció por completo. En el seguimiento posterior (26 meses) se comprobó un descenso progresivo en el porcentaje de estimulación auricular que pasó del 15 al $1,4 \%$. La interrogación del dispositivo confirmó que nunca hubo necesidad de estimulación ventricular.

\section{Discusión}

La endarterectomía carotídea es el tratamiento de elección de los pacientes con estenosis carotídea asintomática significativa $(>60 \%)$, en tratamiento con antiagregantes y estatinas, una esperanza de vida superior a 5 años y baja mortalidad perioperatoria $(<3 \%)^{1}$. La aparición de inestabilidad hemodinámica (hipertensión y/o hipotensión) tras la endarterectomía carotídea es frecuente y parece asociarse a un incremento en la morbimortalidad perioperatoria y en el seguimiento a un año ${ }^{2}$. Sin embargo, aunque la incidencia de bradiarritmias en el postoperatorio precoz es frecuente, estas son transitorias y de buen pronóstico siendo anecdótica la necesidad de estimulación cardiaca permanente ${ }^{3}$.

El síndrome del seno carotídeo cursa con síncopes, generalmente sin pródromos ${ }^{4}$. Suele afectar a ancianos varones, a menudo con historia de enfermedad cardiovascular. Los síncopes se presentan con una respuesta cardioinhibidora (asistolia de $>3 \mathrm{~s}$ ), vasodepresora (caída tensional $>50 \mathrm{mmHg}$ ) o mixta. La causa es desconocida, aunque se distinguen 2 grupos de pacientes: aquellos con procesos cervicales (masas, tumores, cirugías, radioterapia) ${ }^{5}$ y los que aparecen de manera espontánea en relación a compresión mecánica transitoria por corbatas, afeitado o giros del cuello. En nuestro paciente no existía relación con la estimulación mecánica del seno carotídeo. El tratamiento de las formas cardioinhibidoras es el implante de un marcapasos.
Parece evidente desde el punto de vista fisiopatológico que la inflamación del glomus carotídeo provocada por la endarterectomía podría causar un síndrome del seno carotídeo. Sin embargo, la aparición de trastornos del ritmo tras este tipo de cirugía no debe ser muy frecuente. Hasta donde sabemos, este es el segundo caso de implante de marcapasos tras endarterectomía descrito en la literatura ${ }^{6}$.

En el diagnóstico diferencial de los síncopes en este paciente ${ }^{7}$ consideramos otras alternativas como el origen arrítmico primario o el origen neuromediado. La relación temporal que existía entre la endarterectomía carotídea y el inicio de la clínica nos sugería que podía haber una relación causal con la inflamación del glomus carotídeo. La respuesta hipotensiva del paciente al masaje del seno carotídeo derecho también indicaba cierta hipersensibilidad del mismo. El resultado obtenido en el test de atropina reflejaba una buena competencia cronotropa. Por último, el hecho de que los síncopes desapareciesen tras el implante de un marcapasos y que la estimulación auricular fuera disminuyendo progresivamente a lo largo del seguimiento apoyan el diagnóstico de síndrome del seno carotídeo postendarterectomía con predominio de la respuesta cardioinhibidora.

\section{Bibliografía}

1. Tendera M, Aboyans V, Bartelink ML, et al., European Stroke Organisation. ESC Guidelines on the diagnosis and treatment of peripheral artery diseases: Document covering atherosclerotic disease of extracranial carotid and vertebral, mesenteric, renal, upper and lower extremity arteries: The Task Force on the Diagnosis and Treatment of Peripheral Artery Diseases of the European Society of Cardiology (ESC). Eur Heart J. 2011;32:2851-906

2. Tan TW, Eslami MH, Kalish JA, et al., Vascular Study Group of New England. The need for treatment of hemodynamic instability following carotid endarterectomy is associated with increased perioperative and 1-year morbidity and mortality. J Vasc Surg. 2014;59:16-24.

3. Margulies DR, Hestrin MA, Lemus JF, et al. Bradycardia following carotid endarterectomy. Am Surg. 1993;59:578-81.

4. Sutton R. Carotid sinus syndrome: Progress in understanding and management. Glob Cardiol Sci Pract. 2014;2014: $1-8$.

5. Hong AM, Pressley L, Stevens GN. Carotid sinus syndrome secondary to head and neck malignancy: Case report and literature review. Clin Oncol ( $R$ Coll Radiol). 2000;12: 409-12.

6. Ross IB, Guzmán RP. Carotid endarterectomy results in the early years of practice. Surg Neurol. 2001;56:46-52.

7. Moya A, Sutton R, Ammirati F, et al., Task Force for the Diagnosis and Management of Syncope; European Society of Cardiology (ESC); European Heart Rhythm Association (EHRA); Heart Failure Association (HFA); Heart Rhythm Society (HRS). Guidelines for the diagnosis and management of syncope (version 2009). Eur Heart J. 2009;30:2631-71.

Javier Jiménez-Díaz*, Felipe Higuera-Sobrino, María Arántzazu González-Marín y Agustín Camacho-Pedrero

Servicio de Cardiología, Hospital General Universitario de Ciudad Real, Ciudad Real, España 
* Autor para correspondencia. Servicio de Cardiología, Hospital General Universitario de Ciudad Real, C/ Obispo Rafael Torija, s/n, 13005 Ciudad Real, España.

Teléfono: +34926278000, Ext.: 78757.

Correo electrónico: javierjimenezdiaz@wanadoo.es (J. Jiménez-Díaz). http://dx.doi.org/10.1016/j.acmx.2016.07.004

1405-9940/

( 2016 Instituto Nacional de Cardiología Ignacio Chávez. Publicado por Masson Doyma México S.A. Este es un artículo Open Access bajo la licencia CC BY-NC-ND (http: / / creativecommons.org/ licenses/by-nc-nd/4.0/).

\section{Miocardiopatía hipertrófica obstructiva y anemia: causa y consecuencia}

\section{Hypertrophic obstructive cardiomyopathy and anemia: Cause and effect}

\section{Introducción}

La miocardiopatía hipertrófica $(\mathrm{MCH})$ es la miocardiopatía hereditaria más frecuente. Está causada por mutaciones en los genes que codifican las proteínas sarcoméricas, y se caracteriza por una hipertrofia asimétrica del septo interventricular, y distintos grados de obstrucción dinámica del tracto de salida del ventrículo izquierdo (OTSVI) por el movimiento sistólico anterior (SAM) de la valva anterior mitral ${ }^{1}$.
Su diagnóstico se basa en la detección de un aumento del grosor de la pared del ventrículo izquierdo (VI) mediante cualquier modalidad de imagen; incluyendo también el fenotipo de la enfermedad, la presencia de fibrosis miocárdica, las anomalías morfológicas del aparato de la válvula mitral, la función anómala de la microcirculación coronaria y las anomalías electrocardiográficas ${ }^{1}$.

La identificación de la OTSVI es importante en el manejo de los síntomas, y en la evaluación del riesgo de muerte súbita cardíaca. Se sabe que existen causas reversibles que pueden aumentar el gradiente dinámico: fármacos vasodilatadores, disminución de la precarga, ejercicio vigoroso, anemia..., por lo que se deben evitar dichas causas. El tratamiento farmacológico de elección es el uso de betabloqueantes ${ }^{1,2}$. Presentamos el caso de un paciente con $\mathrm{MCH}$ obstructiva severa y anemia poco habitual.
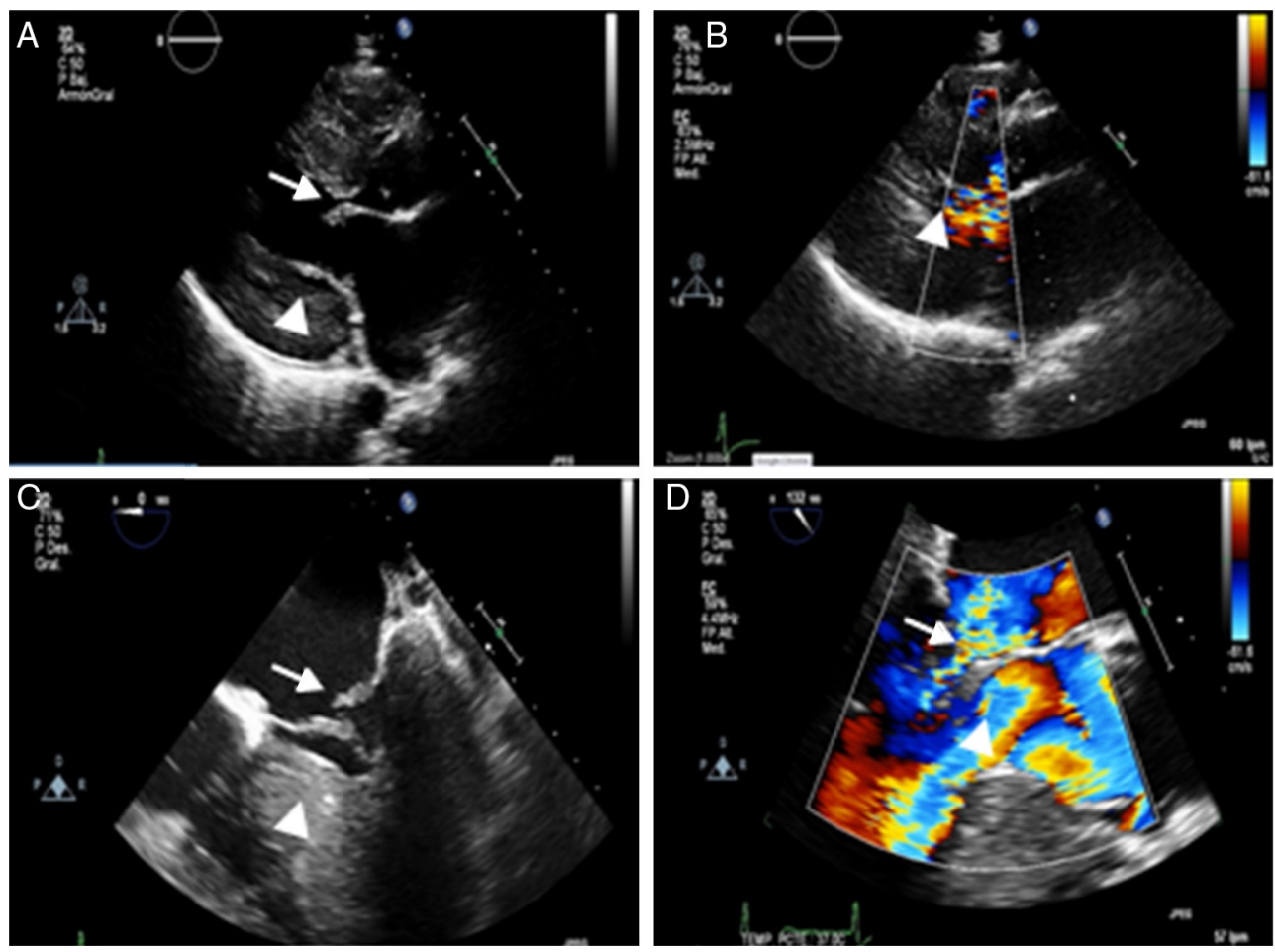

Figura 1 Ecocardiograma transtorácico (ETT) y transesofágico (ETE): A) Plano paraesternal eje largo se objetiva un vi con hipertrofia severa (punta de flecha) y diámetro disminuido, con velo mitral anterior algo engrosado (flecha). B) Plano paraesternal eje largo en sístole, con vi con colapso medioapical por la hipertrofia severa y con flujo turbulento tanto a nivel mitral como en el tracto de salida del vı (punta de flecha). C) ETE en proyección 4 cámaras donde destaca la hipertrofia ventricular (punta de flecha) con válvula mitral engrosada con prolapso y mala coaptación del velo posterior (flecha). D) ETE en plano 3 cámaras, con insuficiencia mitral severa (punta de flecha) y obstrucción dinámica en el tracto de salida (flecha). 